\title{
The overlap passes a chiral dynamical test in two dimensions. *
}

\author{
Yoshio Kikukawa $^{\mathrm{\dagger} \dagger}$ and Herbert Neuberger ${ }^{\mathrm{a} \ddagger}$ \\ ${ }^{a}$ Rutgers University, Department of Physics and Astronomy, Piscataway NJ08855.
}

\begin{abstract}
A certain $U(1)$ model in 2 dimensions, describing four right handed unit charged Weyl fermions interacting with one doubly charged left handed Weyl fermion, is exactly soluble and has massless Majorana-Weyl composites. Instanton induced fermion number violation is essential for 't Hooft anomaly consistency. The associated 't Hooft vertex can be analytically computed in the continuum, including finite size corrections. This number is reproduced in a dynamical simulation employing the overlap.
\end{abstract}

This contribution is based on joint work [1 3 , but is written in first person, close to the oral delivery by the presenter.

In this talk I focus on continuum physics and on the central role played by gauge field topology in our model. I shall introduce a certain one-point function, a dimensionless vertex $V(x)$, whose expectation value has been analytically computed, finite size effects included. $\langle V\rangle$ has been reproduced in a full dynamical simulation based on the overlap.

The model in the abstract could be called the 11112 model, or the 412 model. It is in two (Euclidean) dimensions, has gauge group $U(1)$, and the following fermionic matter content: 4 RW fermions with $q=1\left(\chi_{i}, i=1,2,3,4\right)$ and one $\mathrm{LW}$ fermion with $q=2(\psi)$. R(L)W means right(left) moving Weyl fermions. The model can be generalized to the $S U(2)$ gauge group with matter content of 2 RW of $I=\frac{1}{2}$ and 1 LMW of $I=1$. MW means Majorana-Weyl.

The model is interesting because:

- It is definitely chiral (I mean it is not vector like in disguise - this is evident as it has an odd total number of $\mathrm{W}$ fermions).

- There is confinement, a trivial consequence of two dimensional electrodynamics.

- The gauge field configurations fall into separate topological classes and there are instanton solutions on a toroidal space-time.

\footnotetext{
*Talk at Lattice '97, Edinburgh, July 22 - 26, 1997.

†Permanent address: Department of Physics, Kyoto University, Kyoto 606-01, Japan.

${ }^{\ddagger}$ Presenter; Research supported in part by the DOE under grant \#DE-FG05-96ER40559.
}

- The model is gauge invariant by nontrivial anomaly cancelation; $2^{2}=1^{2}+1^{2}+1^{2}+1^{2}$.

- The massless spectrum consists of composite "baryons". These "baryons" are neutral, made out of two distinct $\chi_{i}$ 's and one anti$\psi$. It follows that they are massless right movers. Naively, one would guess that the baryons are $\mathrm{W}$ fermions, but this is wrong: The anti-baryon can mix with the baryon despite the difference in fermion numbers and the baryons are MW. (The particular 11112 charge values ensure a particle interpretation of the massless sector of the theory. Generically, the massless sector of the full theory would be described by a conformal two dimensional theory admitting no particle interpretation.)

- The model contains a nontrivial example of 't Hooft matching. The symmetry in question is a global $S U(4)$ acting on the $\chi_{i}$ 's which transform as a $\underline{4}$, the fundamental representation. Under the same $S U(4)$ the baryons transform in the six dimensional (antisymmetric) representation. 't Hooft matching requires the baryons to be $\mathrm{MW}$ fermions, not $\mathrm{W}$ fermions.

- The model is exactly soluble. 't Hooft matching only provides a consistency check on a proposed particle spectrum. Here the particle spectrum can be directly found. For the spectrum to come out correctly, in any regularization, it is imperative that fermion number violation of the exactly right amount occur in the continuum limit. 
- Exact solubility extends to a finite torus. We want to get real numerical agreement for some quantity measuring fermion number violation - if agreement is not found the spectrum will not be reproduced. It would be quite difficult to get good numbers if we also had to fight numerically against finite size effects, since the theory has massless particles. It is therefore almost a necessary requirement in practice, at least at the beginning, when suspicions about any proposal ought to be high, to be able to provide analytic results that take finite volume effects fully into account in the continuum.

- In the continuum the chiral determinant is positive. The road to simulations of truly chiral models is paved with difficulties. It is enough not to surmount one and a serious positive test gets out of reach. A major obstacle to Monte Carlo simulation is the expected complex integration measure. Thankfully, here, in the continuum, on the torus, there is a set of boundary conditions that make the product of chiral determinants positive. On the lattice this is not exactly true, but the phase is therefore just a lattice artifact, and one can get away without importance sampling, including the phase (and most of the real part) in the observable. The positivity is a special property of our model, but without it, full dynamical simulation would be much more difficult. As stressed before, there is no doubt that the model is genuinely chiral.

The dimensionless fermion number violating 't Hooft vertex we chose to work with is given by:

$V(x)=\frac{\pi^{2}}{e_{0}^{2}} \chi_{1}(x) \chi_{2}(x) \chi_{3}(x) \chi_{4}(x) \bar{\psi}(x)(\sigma \cdot \partial) \bar{\psi}(x)$.

We derived an exact analytical expression for $\langle V\rangle_{t \times l}$ on a torus of sides $t$ by $l$. Our simulation parameters need $\langle V\rangle_{t \times l}$ at $t=l=\frac{3 \sqrt{\pi}}{2 e_{0}}$ ( $e_{0}$ is the continuum gauge coupling) and there $\langle V\rangle_{t \times l}=0.0389$, substantially smaller than the value at infinite volume.

The objective of our numerical work is to reproduce this last continuum number on the lattice.
By simple arguments we expect convergence as $a^{2}$, where $a$ is the lattice spacing, defined as the ratio between the physical size of the lattice and the number of sites used, $L^{2}$. One therefore expects convergence as $1 / L^{2}$ towards the continuum result. Within the statistical accuracy of our results this indeed happens (see figure).

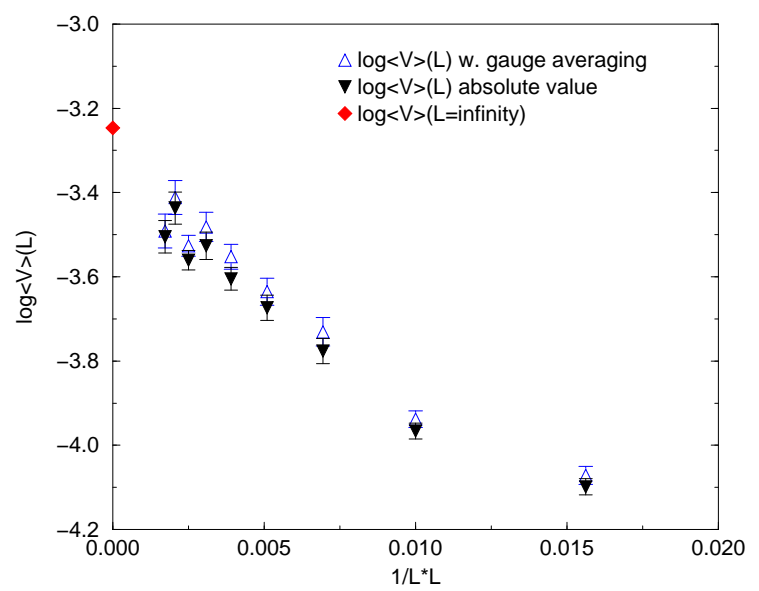

We worked with one point functions, since these typically have the least statistical noise and it was important for us to get good numerical agreement. There is one possible source of contamination that we cannot eliminate cleanly, namely the generation of a marginal Thirring coupling. Such couplings were seen to be generated in single and multi flavored vector like Schwinger models regularized by the overlap [4], so their presence here is not a consequence of chirality. The evidence that the numerical effects we observed in the vector like case (where simulations are much easier) indeed were Thirring couplings is based on the dependence on the number of flavors and the lattice spacing. To avoid the generation of Thirring couplings we tune a free mass parameter in the overlap. This parameter is an extra ultraviolet cutoff, beyond the lattice spacing.

Let me end with some closing comments of a more general nature. Below are three very 
basic questions that would appeal to any particle physicist, working on lattice field theory or not. A serious proposal for a general scheme to non-perturbatively regularize chiral gauge theories should answer positively all three of them.

1. Does the proposal hinge on anomaly cancelation? This excludes any "quenched" tests or mean field arguments: one loop fermion physics is essential.

2. Does the proposal collapse to a simple clear lattice formulation when applied to a vector like theory? Too often is it unclear whether new proposals work at all in the vector like case.

3. Does the proposal address directly and explicitly instanton induced violations of otherwise good conservation laws?

It is inconceivable to me that a general scheme could work in four dimensions but fail in two. Thus, I would add to the above minimum a fourth question: Does the proposal work in a nontrivial two dimensional case, similar to the 412 model above? We have done a certain amount of ground work in the continuum which could be useful also to others who would choose specifically the 412 model as a test case for their proposal.

The overlap should not be the single way to regulate chiral fermions. However, at this juncture in time, it has established a sort of a benchmark for what a proposal can do, and this progress should not be ignored.

I think there are two major levels at which there are open issues. At the most fundamental level lies the question of existence of an asymptotically free chiral gauge theory (this would include the supersymmetric case). Some (e.g. [5]) conjecture these theories do not exist. Others (e.g. [6]) try to rigorously prove they do. The fundamental issue is sharp, a question in mathematical physics, independent of what Nature chose to do. The second level is really relevant only if one believes that there do exist asymptotically free chiral gauge theories, since one cannot prove a negative, and the collection of failures is large enough already. The issue is what constitutes, by present day standards, a credible, valid approach. I claim that a positive answer to the three questions is a minimum requirement. The overlap satisfies this minimum requirement and went beyond it. I invite other proposals to attain a similar stage.

Having emphasized more stringent requirements I should also stress that one should not err in the opposite direction. While numerical QCD research is of potential importance phenomenologically, it would be a mistake to require workers on lattice chiral gauge theories to produce now results at the lattice QCD level, because this would simply shut down the subfield and any progress would stop. QCD work might [7], and already has [8], benefited from research on lattice chiral gauge theories. And we should never forget that the problem of regularizing chiral gauge theory is of a fundamental importance exceeding that of determining accurate numerical predictions of QCD.

\section{REFERENCES}

1. R. Narayanan. H. Neuberger, Phys. Lett. B 393, (1997) 360.

2. Y. Kikukawa, R. Narayanan, H. Neuberger, Phys. Lett. B 399, (1997) 105.

3. Y. Kikukawa, R. Narayanan, H. Neuberger, hep-lat/9705006.

4. R. Narayanan, H. Neuberger, P. Vranas, Nucl. Phys. B (Proc. Suppl.) 47, 596-598 (1996).

5. I. Montvay, hep-ph/9708269.

6. G. 't Hooft Phys. Lett. B349 (1995) 491.

7. H. Neuberger, hep-lat/9707022.

8. R. Narayanan, P. Vranas, hep-lat/97020005. 